\title{
20 Jahre Suburbanisierung der Bevölkerung
}

\author{
Zur Stadt-Umland-Wanderung in westdeutschen Großstadtregionen
}

\section{0 years of Suburbanisation of the Population}

Migration from the city into suburban areas oder urban fringe areas in West German metropolitan regions

\section{Kurzfassung}

Jedes Jahr verlieren die großen deutschen Städte durch die Suburbanisierung Einwohner an ihr Umland. Die Untersuchung von sieben Großstädten ergibt zwischen 1981 und 2000 einen jährlichen negativen Wanderungssaldo von etwa $0,5 \%$ an der Gesamtbevölkerung. Somit sind in 20 Jahren ca. $10 \%$ der Stadtbevölkerung in das Umland abgewandert. Die Suburbanisierung ist ein wesentlicher Grund für das Verhältnis der einwohnerbezogenen Baufertigstellungen, wobei die Kernstadt gegenüber dem Umland mit einem Verhältnis von 1:1,7 deutlich weniger Wohnungen auf den Markt bringt. Im Prozess der Suburbanisierung spielt der Gebäudetyp des so genannten Ein- und Zweifamilienhauses eine besondere Rolle. Die Untersuchung macht in allen Städten die höhere Attraktivität des Umlandes und das knappere Angebot an Baugrundstücken in den Kernstädten für die Errichtung von Ein- und Zweifamilienhäusern deutlich: So liegen die einwohnerbezogenen Baufertigstellungen vier bis fünf Mal höher als im Zentrum.

\section{Abstract}

Every year the major German cities are losing inhabitants through the suburbanisation of their urban fringe. The study of seven major cities reveals an annual negative migration balance of about $0.5 \%$ of the total population between 1981 and 2000. Thus approximately $10 \%$ of the urban population migrated to the surrounding countryside in 20 years. Suburbanisation is an essential reason for the ratio of population-related housing completions, in which the inner city brings considerably fewer dwellings onto the marketwith a ratio of 1:1.7 - in comparison with the urban fringe. The building type of so-called "single-family" and "two-family" houses plays a special role in the process of suburbanisation. The study illustrates the greater attractiveness of the urban fringe and the scarce availability of building sites in the inner cities for the construction of single-family and two-family houses: as a result, the population-related housing completions in urban fringe areas are four to five times higher than in the city centre.

\section{Suburbanisierung in Deutschland}

„Ziehen Sie aufs Land, dann haben Sie es weiter in die Stadt". Dieser Werbeslogan eines deutschen Automobilherstellers bringt das tägliche Pendeln von Millionen Menschen über immer größere Distanzen ganz gut auf den Punkt. ${ }^{1}$ Es ist zwar nicht anzunehmen, dass der von der Werbung versprochene tägliche Fahrspaß der Hauptgrund für das Fortziehen vieler Haushalte in immer entferntere Umlandgebiete ist. Jedoch wurde erst durch die Individualmotorisierung eines Großteils der Bevölkerung und die Schaffung einer entsprechenden Infrastruktur die Grundlage für die dispersen Siedlungsstrukturen in den heutigen Ausmaßen geschaffen. 
$\mathrm{Zu}$ den Wanderungsstrukturen und -motiven liegen mehrere empirische Untersuchungen vor, die auf Befragungen bei in das Umland abgewanderten bzw. in die Stadt zuziehenden Personen basieren. Ohne an dieser Stelle auf Detailergebnisse der ausgewerteten Studien $^{2}$ einzugehen, sind folgende Grundmuster der Zu- bzw. Fortziehenden festzustellen.

Die in die Stadt Zuziehenden sind in der Regel jüngere Einpersonenhaushalte mit geringem bis mittlerem Einkommen, die zur Ausbildung bzw. zum Start in das Berufsleben in die Stadt ziehen und zu einem höheren Anteil als bei den Fortziehenden ihren neuen Wohnort nur als befristete Übergangslösung ansehen. Das bedeutendste Wanderungsmotiv ist die Nähe zum Ausbildungs- bzw. Arbeitsort. Zusätzlich werden die räumliche Nähe zu Freunden und Bekannten, sowie zu kulturellen Angeboten und Freizeiteinrichtungen genannt.

Die Fortziehenden sind in der Regel Mehrpersonenhaushalte. Dabei handelt es sich um kinderlose Paare oder Paare mit Kind bzw. Kindern aus mittleren Altersgruppen, die erwerbstätig sind und über höhere Haushaltseinkommen verfügen. Wesentliche Wanderungsmotive sind die Verbesserung der unmittelbaren Wohnsituation und des Wohnumfeldes. Dabei werden die besseren Wohnbedingungen des Umlandes mit der großstädtischen Infrastruktur des Zentrums (z. B. Theater, Museen) kombiniert. Die damit verbundenen Konsequenzen wie erhöhte Mobilitätserfordernisse werden in Kauf genommen.

Diese Darstellung entspricht weitgehend den bekannten klassischen Wanderungsmustern, die klischeehaft als „Abiturient geht zum Studieren in die Stadt“ bzw. „Familie sucht ein Haus im Grünen“ umschrieben werden können. In der aktuellen wissenschaftlichen Diskussion ${ }^{3}$ wird vermutet, dass sich die Wanderungsstrukturen in Zukunft immer stärker ausdifferenzieren werden. Möglicherweise werden in Zukunft nicht mehr nur die Familie in der Gründungsphase den Schwerpunkt der Stadt-Umlandwanderer bilden, sondern auch das kinderlose Paar oder die allein stehende Person. Dabei bestimmt häufig das Angebot die Nachfrage. So kommen mittlerweile Einfamilienhäuser auf den Markt (auch zur Miete), die von den Suburbanisierern der 60er und 70er Jahre erstellt worden sind.

Die Realisierung einer Wanderungsentscheidung hängt nicht zuletzt an vielen individuellen Einzelentscheidungen, bei denen die (Wunsch-)Vorstellungen an die Wohnsituation und das Wohnumfeld mit der realen Situation sowie den - vielfach finanziel! limitierten - Möglichkeiten zur Veränderung „abgeglichen“ werden müssen: So kam bei einer Umfrage nach dem präferierten Haustyp mit weitem Abstand das freistehende Ein- bzw. Zweifamilienhaus bei $73 \%$ (West) bzw. $66 \%$ (Ost) aller Befragten als ihr Wunsch heraus. In der Realität lebten aber lediglich $34 \%$ (West) bzw. $27 \%$ (Ost) in diesem Haustyp. ${ }^{4}$

Insgesamt ist davon auszugehen, dass sich im Zentrum und im Umland sowohl die Nachfrage als auch das Angebot von Wohnraum in verschiedenen Gebäudetypen bzw. Baugrundstücken in attraktiven Lagen stärker als bisher nach qualitativen Aspekten weiterentwickeln werden. ${ }^{5}$ Dies zeigen auch die 34,4 Mio. bestehenden Bausparverträge bei 11 Landesbausparkassen und 17 privaten Bausparkassen mit einem Gesamtvolumen von $678 \mathrm{Mrd}$. $€{ }^{6}$ Auch wenn viele Spareinlagen nicht direkt für die Schaffung von Wohneigentum verwendet werden und die derzeitige Diskussion um staatliche Förderungen einige Veränderungen erwarten lassen, ist von einem weiteren Kapitalzufluss in Wohnimmobilien zur qualitativen Erneuerung des Bestandes an attraktiven Standorten auszugehen.

Die aus der Suburbanisierung resultierenden Auswirkungen auf das Verhältnis von Stadt und Umland hat das Bundesamt für Bauwesen und Raumordnung im aktuellen Raumordnungsbericht ${ }^{7}$ beschrieben. So müssen sich die deutschen Stadtregionen mit diversen Problemlagen der Bevölkerungssuburbanisierung mehr oder weniger intensiv auseinander setzen:

- Die vielfach unkoordinierte Ausweisung von Wohnund Siedlungsflächen im Umland führt zu einer ungeordneten Raumentwicklung und Zersiedelung der Landschaft.

- Die räumlichen Distanzen zwischen Wohnungen, Arbeitsplätzen, Versorgungs- und Infrastruktureinrichtungen steigen weiter und erzeugen erhebliche Verkehrsprobleme.

- Diverse Infrastruktureinrichtungen der Städte sind nicht mehr ausgelastet. Das Umland baut diese parallel neu auf und ,entkoppelt" sich zunehmend von der Kernstadt.

- Die Kernstädte verlieren durch den Wegzug von Haushalten mit höheren Einkommen einen wichtigen Teil ihrer Steuereinnahmen.

- In manchen Stadtteilen der Zentren konzentrieren sich soziale Probleme, die u.a. mit der Suburbanisierung und dem Fortziehen der Mittelschichten zusammenhängen.

Die Auflistung zeigt einige Felder regionaler Problemlagen, die nicht zuletzt zu politischen Diskursen zwischen den Städten und den Umlandregionen geführt haben. ${ }^{8}$ Auf Grund der räumlichen Verflechtungen werden Stadt und Umland in zunehmender Weise nicht mehr als gegensätzliche Einheiten, sondern als eine Stadtregion betrachtet. Relativ weitgehend sind die Kooperationsformen in Hannover und Stuttgart. Aber auch andere Regionen, vor allem solche mit 
einem hohen regionalen Entwicklungsdruck wie München $^{9}$, denken über derartige Initiativen nach.

Die wissenschaftliche Diskussion ${ }^{10}$ zum Thema Suburbanisierung orientierte sich in den vergangenen Jahrzehnten vielfach an der Intensität und der Richtung der Stadt-Umland-Wanderungen. Bedingt durch vielfältige Einflussfaktoren, wie die allgemeine konjunkturelle Lage, den Zuzügen aus dem Ausland oder dem generativen Verhalten der Bevölkerung unterliegen die Suburbanisierungstendenzen bestimmten Zyklen. So drehte sich Ende der 80er Jahre die Diskussion schon einmal um die „Reurbanisierung der Bevölkerung?" als die Suburbanisierungsverluste in den Jahren zuvor immer mehr zurückgingen. Im Gegensatz dazu - und wie in diesem Beitrag anhand von Zeitreihen gezeigt wird - konnte in den 90er Jahren allerdings eine deutliche Zunahme der Umlandwanderungen beobachtet werden, die nicht zuletzt durch den Fall des „Eisernen Vorhangs“ und durch starke Ost-West-Wanderungen begleitet wurden. Da viele Städte in den 90er Jahren auch insgesamt deutlich an Einwohnern verloren haben, ist die Suburbanisierung der Bevölkerung umso mehr in das Bewusstsein von Städten und Regionen geraten. Seit 1999 ist wieder eine gewisse Gegentendenz - Einwohnerzuwachs in den Städten und Abschwächung der Suburbanisierungsverluste - festzustellen.

Ziel dieses Beitrags ${ }^{12}$ ist es, das Ausmaß der StadtUmland-Wanderung in einem Regionalvergleich über einen möglichst langen Zeitraum zu analysieren und einzuschätzen. Anhand der zugänglichen Statistiken werden Gemeinsamkeiten und Unterschiede in den langfristigen Entwicklungstrends zwischen den ausgewählten Agglomerationsräumen Bremen, Hamburg, Hannover, Frankfurt am Main, Nürnberg, Stuttgart und München herausgearbeitet. Dabei wird neben der Suburbanisierung der Bevölkerung der Schwerpunkt auf die Bauaktivitäten in diesen Großstadtregionen gelegt. Um die zeitliche Entwicklung nachvollziehen zu können, werden Zeitreihenanalysen über 20 Jahre durchgeführt. Abschließend wird eine Einschätzung der empirischen Erkenntnisse hinsichtlich ihrer regionalpolitischen Implikationen und der Fortentwicklung der Suburbanisierungsdebatte gegeben.

\section{Auswahl der Untersuchungsregionen}

Im Rahmen dieser Analyse werden alle solitär gelegenen deutschen Großstädte über 400000 Einwohner in die Untersuchung einbezogen. Vergleichbare Daten sind für einen Zeitraum von 20 Jahren (1981-2000) aus der Wanderungsstatistik der Statistischen Landesämter verwendet worden. Auf Grund des langen Beobachtungszeitraums von zwei Jahrzehnten wird auf eine
Betrachtung von Berlin, Dresden und Leipzig verzichtet. Ebenso werden die großen Städte des Verdichtungsraumes an Rhein und Ruhr (Köln, Essen, Dortmund, Düsseldorf und Duisburg) wegen ihrer starken räumlichen Verflechtungen untereinander nicht in die vergleichende Analyse einbezogen. Es verbleiben demnach sieben solitär gelegene Großstädte: Bremen, Hamburg, Frankfurt am Main, Hannover, Stuttgart, Nürnberg und München. Als räumliche Abgrenzung des Umlandes wird in der Regel auf bestehende Raumabgrenzungen zurückgegriffen, u. a. um langfristig vorliegende Datensätze nutzbar zu machen. Schließlich wird in diesem Beitrag der Versuch unternommen, die regionale Entwicklung möglichst jährlich, lückenlos und weit zurück reichend in einer einheitlichen Zeitreihenanalyse gegenüberzustellen.

Abbildung 1 zeigt einen Überblick der untersuchten Städte. Über die mit Abstand größte Fläche verfügt Hamburg mit $755,33 \mathrm{~km}^{2}$. Es folgen Bremen $\left(326,55 \mathrm{~km}^{2}\right)$ und München $\left(310,59 \mathrm{~km}^{2}\right)$. Die kleinste Stadtfläche weist Nürnberg mit $186,37 \mathrm{~km}^{2}$ auf. Hamburg (ca. 1,7 Mio.) und München (ca. 1,2 Mio.) verfügen als „Millionenstädte“ über die meisten Einwohner der sieben analysierten Zentren.

Die untersuchten Städte weisen im Durchschnitt eine Verdichtung in Höhe von 2626 Einwohnern je km² auf. Zwischen den Städten bestehen jedoch deutliche Unterschiede. So ist das Stadtgebiet von München mit $3897 \mathrm{EW}$ je $\mathrm{km}^{2}$ am dichtesten besiedelt. Etwas über dem Durchschnitt aller Städte liegt Stuttgart mit 2816 EW je $\mathrm{km}^{2}$. Es folgen die Zentren Nürnberg (2 621), Frankfurt (2 604), Hannover (2 524) und Hamburg (2 271). Mit 1652 Einwohnern je $\mathrm{km}^{2}$ ist Bremen mit Abstand die Stadt mit der geringsten Einwohnerdichte.

Die sieben untersuchten Städte grenzen ihr Umland nicht nach einer einheitlichen Methode ab. So zieht Bremen einen Kreis von $30 \mathrm{~km}$ um den Marktplatz und erhält somit - im Gegensatz zu den anderen untersuchten Städten - eine gemeindescharfe Abgrenzung mit 23 Gemeinden und sieben Samtgemeinden. Die anderen Regionen setzen sich aus Kreisgrenzen zusammen. In der Regel bestehen diese aus den unmittelbar angrenzenden Kreisen bzw. darüber hinaus aus jenen Kreisen, die große Verflechtungsbeziehungen (z.T. als regionalpolitischer Verbund organisiert) mit der Kernstadt aufweisen.

Die Größe der Umlandregionen schwanken zwischen dem Umland von Hamburg (6 $\left.548 \mathrm{~km}^{2}\right)$ und dem von Hannover $\left(2086 \mathrm{~km}^{2}\right.$ ). Die größte Verdichtung einer Umlandregion ist mit 589 Einwohnern je $\mathrm{km}^{2}$ im Stuttgarter Raum zu sehen. Wie bei den Zentren auch liegt die geringste Verdichtung der analysierten Umlandregionen in Bremen. Dort leben lediglich 163 Einwohner auf einer Fläche von einem $\mathrm{km}^{2}$. 
Abbildung 1 Raumabgrenzung und Einwohnerdichte am 31. Dezember 2000
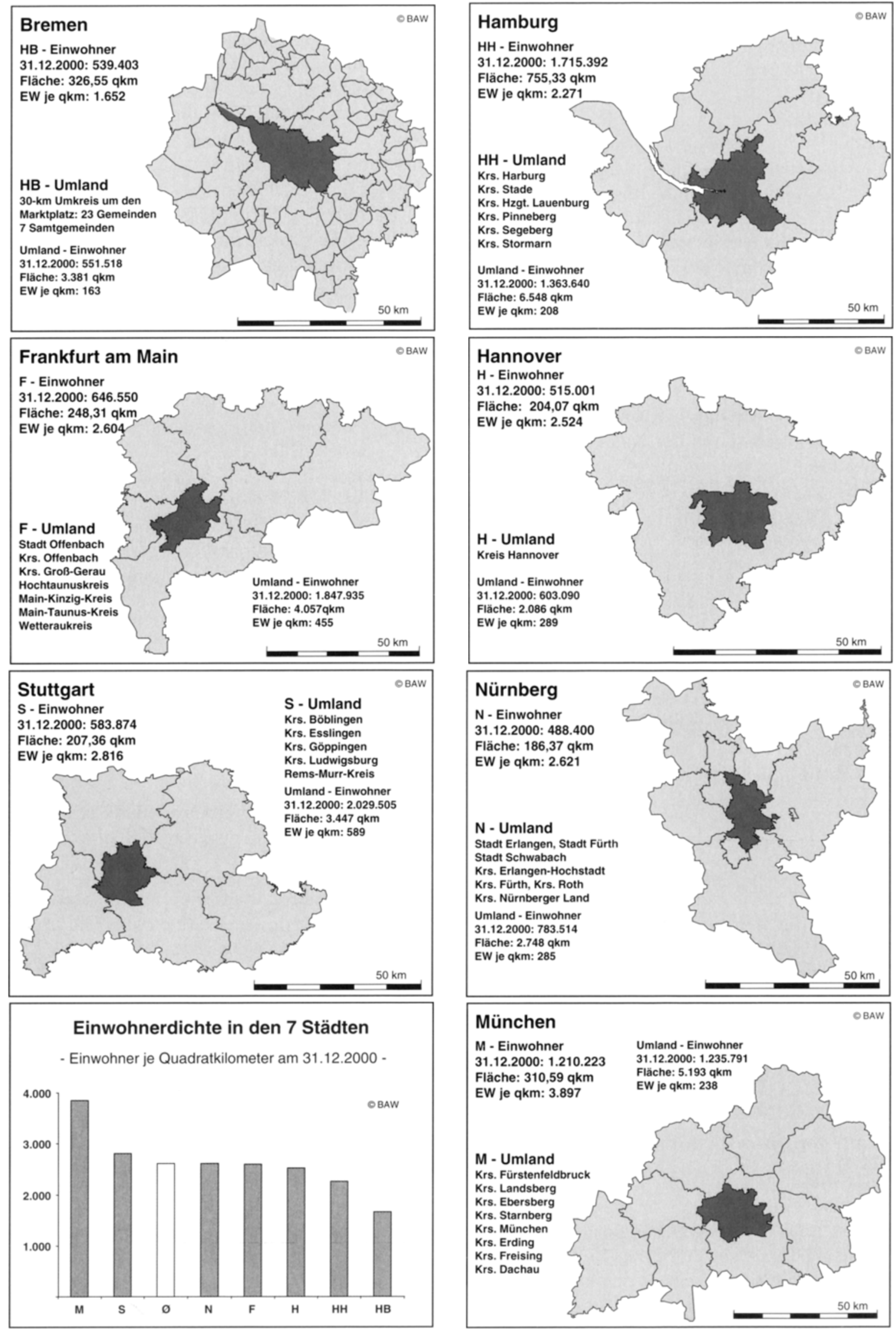

Quelle: Statistisches Bundesamt, Statistische Landesämter, Statistisches Jahrbuch deutscher Gemeinden, eigene Berechnungen 


\section{Suburbanisierungstendenzen in solitär gelegenen Großstadtregionen}

Die folgenden empirischen Erkenntnisse basieren auf Sonderauswertungen für jeweils 20 Beobachtungszeitpunkte, die von den Statistischen Landesämtern zur Verfügung gestellt oder in Statistischen Jahrbüchern veröffentlicht worden sind. ${ }^{13} \mathrm{Um}$ eine einheitliche Datengrundlage sicherzustellen, sind in diese Untersuchung ausschließlich Wanderungsdaten der amtlichen Statistik bzw. Daten der Statistischen Landesämter eingegangen. ${ }^{14}$

Im Ergebnis werden grundsätzliche Gemeinsamkeiten in den Entwicklungsverläufen der Stadt-Umland-Wanderungen zwischen den Städten sichtbar. So gibt es in 20 Jahren und sieben Städten nicht einen einzigen positiven Saldo aus $\mathrm{Zu}$ - und Fortzügen zu Gunsten eines Zentrums. Vielmehr sind die Gewinner der Entwicklung überall die umliegenden Gebiete.

Insgesamt sind im Beobachtungszeitraum 533142 Einwohner per Saldo an das Umland der Städte „verloren" gegangen. Wegen der Stadtgröße nicht verwunderlich, mussten die höchsten absoluten Verluste die Städte München mit 137372 (per anno 6 869) und Hamburg mit 133185 (p.a. 6 659) Einwohnern hinnehmen. Vergleichsweise gering fallen demgegenüber die Einwohnerverluste der Jahre 1981-2000 aus der Sicht des Zentrums in Nürnberg mit 36966 (p.a. 1848 ), Hannover mit 42290 (p.a. 2115 ) und Bremen mit 52616 (p.a. 2 631) Einwohnern aus.

Damit die Entwicklungsverläufe der Suburbanisierungstendenzen miteinander zu vergleichen sind, werden die Salden der Stadt-Umland-Wanderungen auf die jeweiligen Einwohnerzahlen der Städte bezogen. Die empirischen Ergebnisse für die Jahre 1981-2000 sind der Abbildung 2 zu entnehmen.

Auf 100000 Einwohner bezogen, verlieren alle sieben Zentren pro Jahr durchschnittlich 470 Einwohner an das Umland. Die größten durchschnittlichen Bevölkerungsverluste durch die Suburbanisierung müssen demnach Frankfurt mit 588 und München mit 555 je 100000 Einwohner und Jahr hinnehmen. Stuttgart (480) und Bremen (483) weichen nur wenig vom Durchschnitt aller Städte ab. Vergleichsweise geringe einwohnerbezogene Verluste aus der Umlandwanderung sind in Hannover (408), Hamburg (400) und Nürnberg (379) zu sehen.

Zusammengefasst kann also über die 20 Jahre festgehalten werden, dass die Städte jährlich zwischen ca. 0,4 und $0,6 \%$ ihrer Bewohner an das Umland verlieren. In der Summe bedeutet dies etwa für Frankfurt, dass sich im Laufe der Jahre ca. 11,8\% der Gesamtbevölkerung für das Umland als Wohnstandort entschieden haben.
Die Entwicklungstrends für die einzelnen Städte sind in den jeweiligen Zeitreihen der Jahre 1981-2000 enthalten. Neben der Darstellung der negativen Wanderungssalden aus der Sicht der Kommunen ist als Benchmark zudem eine gestrichelte Linie in die Grafiken aufgenommen worden, die den Verlauf der aggregierten Daten aller sieben Städte zeigt. Dadurch werden mögliche Unterschiede einer Stadt zu den allgemeinen Tendenzen der Städtegruppe sichtbar.

Für alle Städte mehr oder weniger stark nachweisbar ist die zweigeteilte Entwicklung der 80er und 90er Jahre. Waren die Suburbanisierungstendenzen zwischen 1981-1990 mit einem durchschnittlichen Einwohnerverlust von 382 je 100000 Einwohner in den untersuchten Städten noch nicht so stark ausgeprägt, so nehmen die Wanderungsverluste aus der Sicht der Zentren seit Anfang der 90er Jahre sprunghaft zu. Zwischen 1991 und 2000 sind die Wanderungsverluste mit 558 pro 100000 Einwohner in der Gruppe der sieben Städte im jährlichen Durchschnitt bedeutend größer.

Von allen Städten musste Frankfurt zwischen 1991 und 2000 die größten jährlichen Wanderungsverluste an das Umland verkraften. Demgegenüber verbuchen Nürnberg und Hamburg in den 90er Jahren die vergleichsweise geringsten Einwohnerverluste in der untersuchten Städtegruppe. Zudem zeichnet sich Hamburg durch relativ geringe Schwankungen in der Stadt-Umland-Wanderung im Gesamtzeitraum aus. Auch Hannover konnte sich die meiste Zeit vom Durchschnitt der Städtegruppe absetzen und hat - relativ betrachtet - weniger Einwohner an das Umland verloren.

Die Entwicklung von Stuttgart und Bremen ist sehr ähnlich verlaufen und pendelt über 20 Jahre hinweg ungefähr im Takt der Benchmark-Städtegruppe. Vergleichsweise hoch waren in Bremen die Einwohnerverluste durch die Stadt-Umland-Wanderung vor allem Anfang bis Mitte der 90er Jahre. Am Ende des Beobachtungszeitraums (1999/2000) ist in fünf der sieben untersuchten Zentren eine Abschwächung der Suburbaniserungsverluste festzustellen. 
Abbildung 2 Stadt-Umland-Wanderung der Bevölkerung 1981-2000
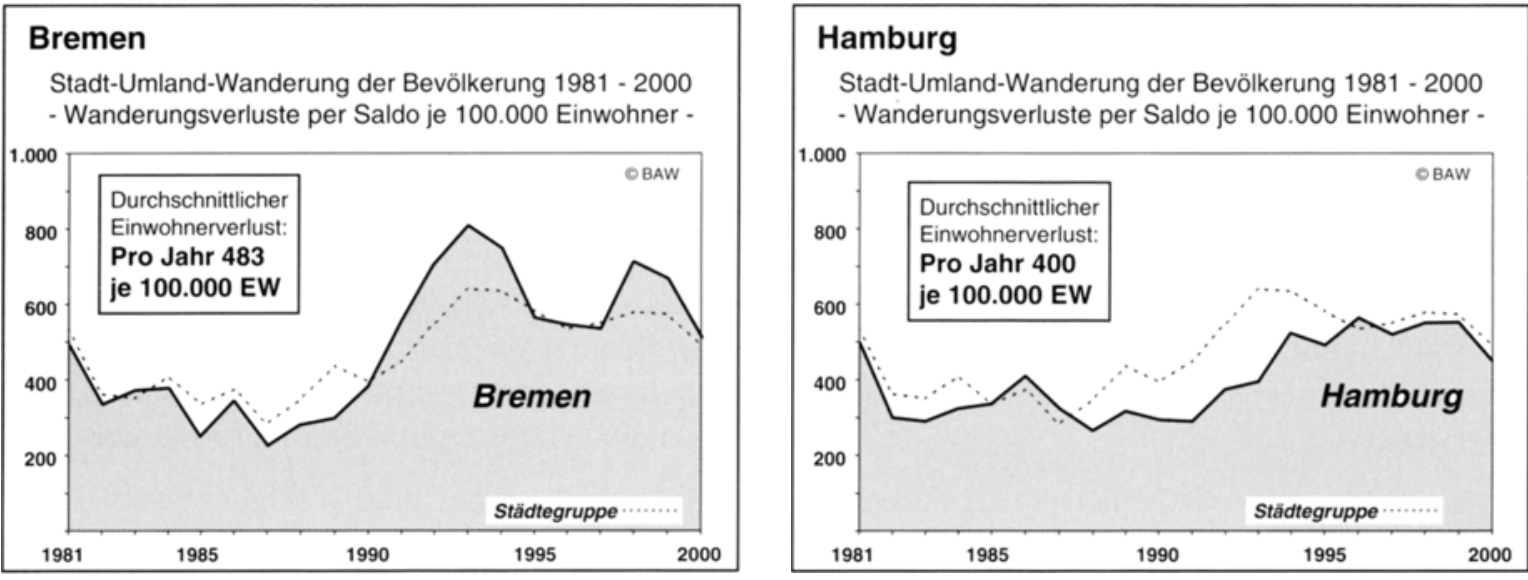

\section{Frankfurt am Main}

Stadt-Umland-Wanderung der Bevölkerung 1981 - 2000

- Wanderungsverluste per Saldo je 100.000 Einwohner -

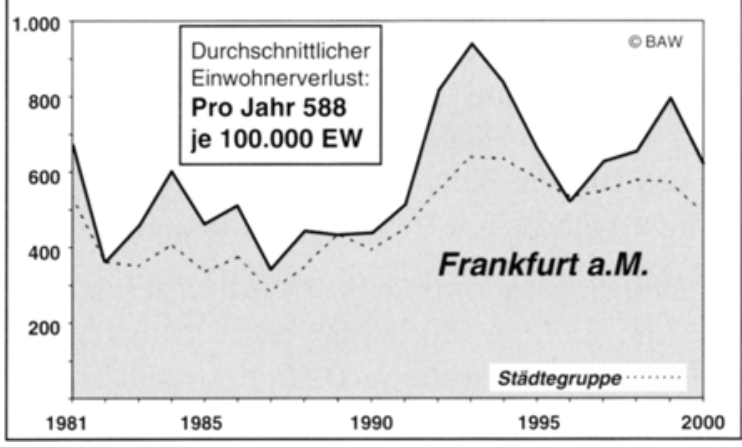

\section{Hannover}

Stadt-Umland-Wanderung der Bevölkerung 1981 - 2000

- Wanderungsverluste per Saldo je 100.000 Einwohner -

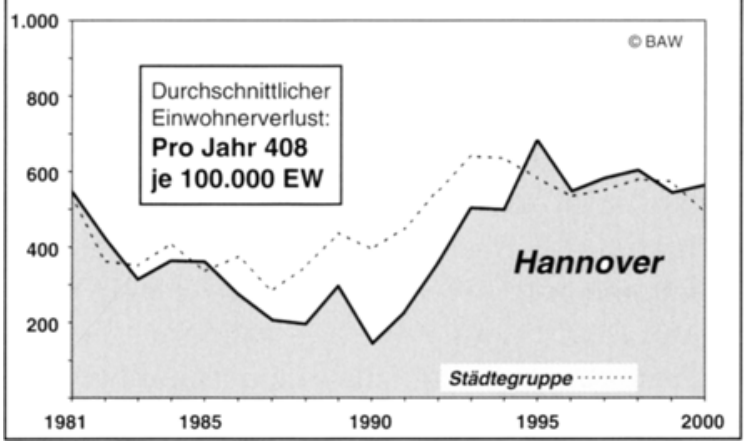

\section{Stuttgart}

Stadt-Umland-Wanderung der Bevölkerung 1981 - 2000

- Wanderungsverluste per Saldo je 100.000 Einwohner .

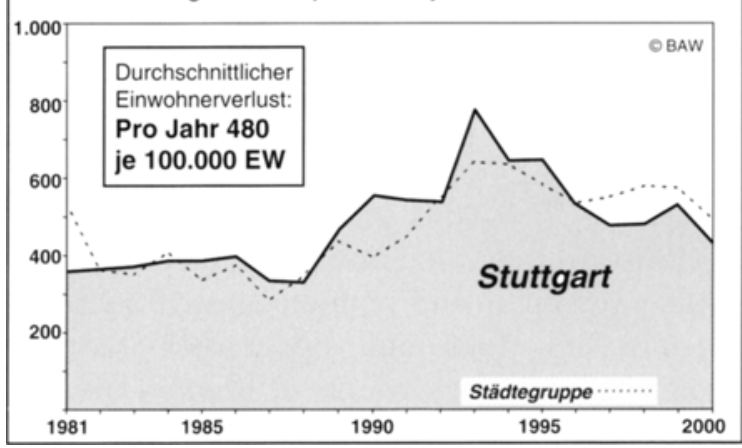

Durchschnittliche Einwohnerverluste pro Jahr

Stadt-Umland-Wanderung der Bevölkerung 1981 - 2000

- Wanderungsverluste per Saldo je 100.000 Einwohner -

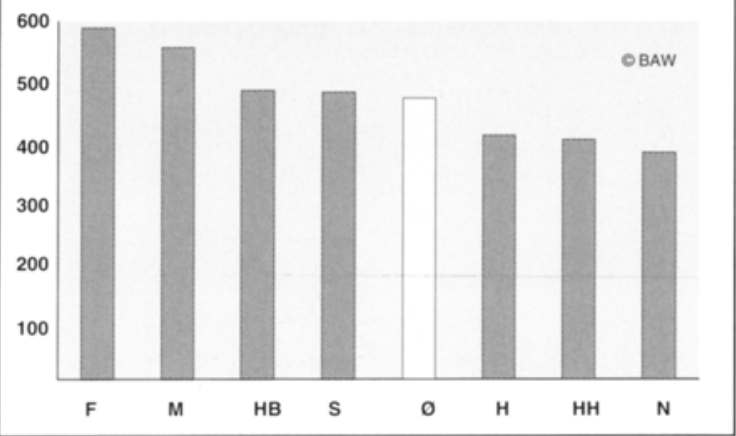

\section{Nürnberg}

Stadt-Umland-Wanderung der Bevölkerung 1981 - 2000

- Wanderungsverluste per Saldo je 100.000 Einwohner .

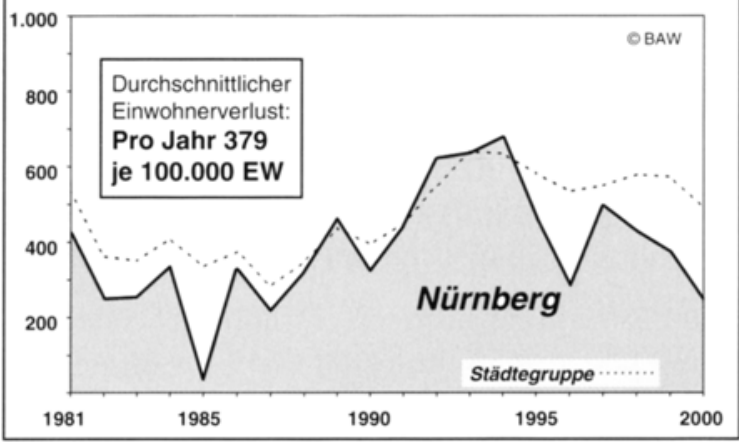

\section{München}

Stadt-Umland-Wanderung der Bevölkerung 1981 - 2000

- Wanderungsverluste per Saldo je 100.000 Einwohner -

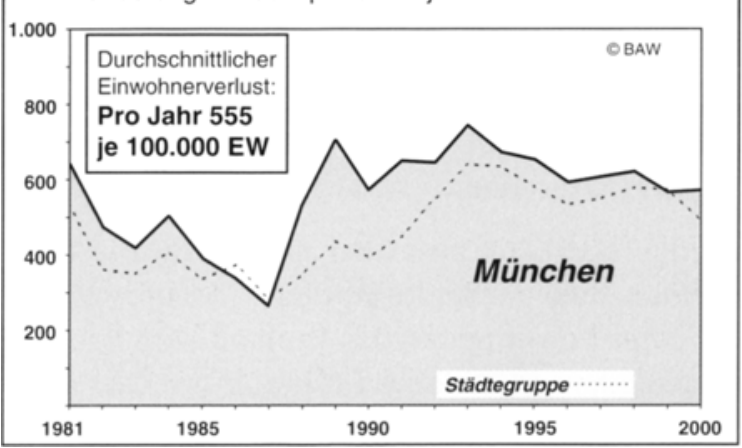

Quelle: Statistisches Bundesamt, Statistische Landesämter, Statistisches Jahrbuch deutscher Gemeinden, eigene Berechnungen 


\section{Baufertigstellungen von Wohnungen und Ein- und Zweifamilienhäusern}

Wie eingangs beschrieben, hat die Verbesserung der individuellen Wohnsituation für die Wanderungsmotive eine besondere Bedeutung. Aus diesem Grund stehen die Baufertigstellungen von Wohnungen und Ein- und Zweifamilienhäusern in Großstadtregionen bei den weiteren Analysen im Mittelpunkt.

Abbildung 3 enthält die Baufertigstellungen von Wohnungen zwischen 1981 bis 2000 je 100000 Einwohner in einer Zeitreihenanalyse. Die Grafiken enthalten den Entwicklungsverlauf der gesamten Region, jeweils getrennt dargestellt nach den Fertigstellungen in der Stadt bzw. im dazugehörigen Umland. Für alle drei Teilräume Region, Stadt und Umland sind die durchschnittlichen Werte aus dem 20-Jahres-Beobachtungszeitraum in der Abbildung angeführt und in der zusammenfassenden Übersicht zum Städtevergleich gegenübergestellt.

Die Untersuchung des Verlaufes der Baufertigstellungen über 20 Jahre zeigt in fast allen Stadtregionen ${ }^{15}$ ähnliche Entwicklungsphasen. An eine Phase hoher Baufertigstellungsaktivitäten zwischen 1981-1985 schließt sich eine Schwächeperiode mit ihrem Tiefpunkt 1986/87 an. Im Anschluss daran ziehen die Fertigstellungen wieder deutlich an und erreichen 1994/ 95 ihren Höhepunkt. Seitdem ist wieder ein rückläufiger Trend zu beobachten. Als Ausnahme dieses zyklischen Entwicklungsverlaufes ist München zu sehen: Über den gesamten Zeitraum von 20 Jahren werden dort die Baufertigstellungen von Wohnungen auf einem sehr hohen und stabilen Niveau gehalten. Alle anderen Regionen haben neben den allgemeinen Trends jeweils individuelle Hoch- und Tiefphasen.

Grundsätzlich lässt sich im 20-Jahre-Verlauf bei der Höhe der Baufertigstellungen eine gewisse Parallelität der Entwicklung zwischen Stadt und Umland feststellen. Es ist zu beobachten, dass bei relativ häufiger bzw. seltener Erstellung von Wohnungen im Zentrum ebenso eine höhere bzw. geringere Bedeutung der Fertigstellungen im Umland zu sehen ist. Dies deutet bereits auf regionale Einflussfaktoren (wie etwa die Entwicklung der Mieten, Bodenpreise, regionale Wirtschaftsentwicklung usw.) hin, die in Stadt und Umland gleichzeitig wirksam werden.

Im gesamten Beobachtungszeitraum wurden in der Gruppe der Stadtregionen insgesamt 1047 Wohnungen pro 100000 Einwohner und Jahr fertig gestellt. ${ }^{16}$ Über diesem Durchschnittswert liegen die Regionen Hamburg (1 057), Nürnberg (1 109), Stuttgart (1 127) und - am deutlichsten - München mit 1308 jährlich erstellten Wohnungen bezogen auf 100000 Einwohner.
Unterhalb des Mittelwertes liegen Bremen (962), Hannover (897) und Frankfurt (871). Damit hat Frankfurt etwa ein Drittel weniger Wohnungen - bezogen auf die Einwohnerzahl - erstellt, als dies in München der Fall war.

Von allen Städten am wenigsten Baufertigstellungen konnte mit lediglich 301 Wohnungen je 100000 Einwohner und Jahr Bremen realisieren. Demgegenüber steht die Stadt München (536) mit fast doppelt so vielen fertig gestellten Wohnungen. Im Umland sticht ebenfalls München mit 772 fertig gestellten Wohnungen je 100000 Einwohner in 20 Jahren hervor. Die geringsten Aktivitäten im Vergleich der Umlandregionen sind demgegenüber im Frankfurter Umland (548) zu sehen.

Abbildung 4 geht in der Analyse der Baufertigstellungen noch einen Schritt weiter und betrachtet nur noch die Erstellung neuer Gebäude nach dem Typ des Hauses. Differenziert werden die Baufertigstellungen neuer Wohngebäude mit einer bzw. zwei Wohnungen. ${ }^{17}$ Zunächst fällt dabei auf, dass - im Gegensatz zur Erstellung von Wohnungen - die Differenzen zwischen den Regionen bei den erstellten Gebäuden deutlich größer ausfallen. So wurden in der Region Bremen über 20 Jahre hinweg im Durchschnitt 429 Ein- und Zweifamilienhäuser pro Jahr und bezogen auf 100000 Einwohner erstellt. Mit lediglich 187 erstellten Gebäuden dieses Haustyps erreicht die Region Frankfurt damit weniger als die Hälfte des Bremer Wertes. Aber auch die Region Stuttgart erreichte nur 233 einwohnerbezogene Fertigstellungen per anno. Es folgen mit deutlich höheren Bauaktivitäten die Regionen Hannover (325), München (367), Nürnberg (372) und schließlich die Region Hamburg, die mit 410 Fertigstellungen von Ein- und Zweifamilienhäusern pro Jahr schon fast den Wert der Region Bremen erreichen kann.

Die Entwicklungsverläufe der Baufertigstellungen von Ein- und Zweifamilienhäusern über 20 Jahre zeigen zunächst weniger starke zyklische Schwankungen als bei der Erstellung von Wohnungen. Zudem fällt auf, dass die Regionen Frankfurt und Stuttgart relativ wenige Baufertigstellungen in der Periode Anfang bis Mitte der 90er Jahre bei den Ein- und Zweifamilienhäusern aufweisen, während die Bauaktivitäten bei den Wohnungen gleichzeitig stark angezogen sind. Beispielsweise wurden auf dem Tiefpunkt im Jahr 1995 in der Stadt Frankfurt gerade noch neun Ein- und Zweifamilienhäuser je 100000 Einwohner erstellt. 
Abbildung 3 Baufertigstellungen von Wohnungen in den Großstadtregionen 1981-2000
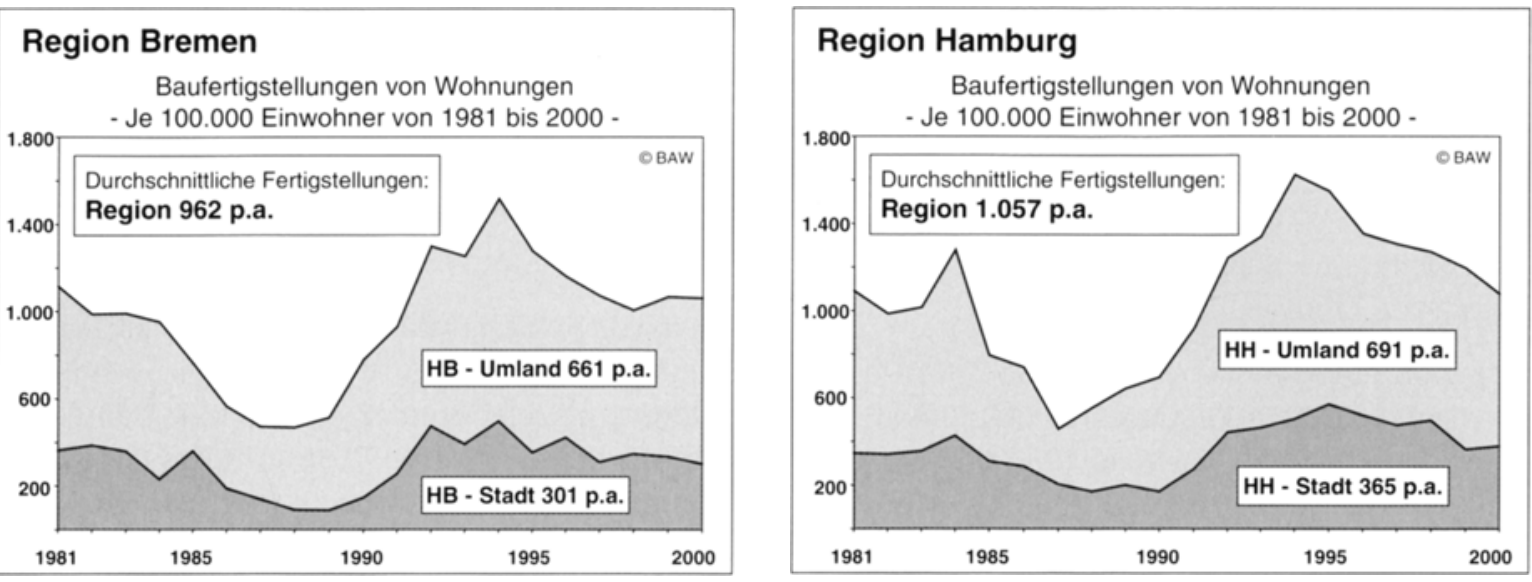

\section{Region Frankfurt am Main}

Baufertigstellungen von Wohnungen

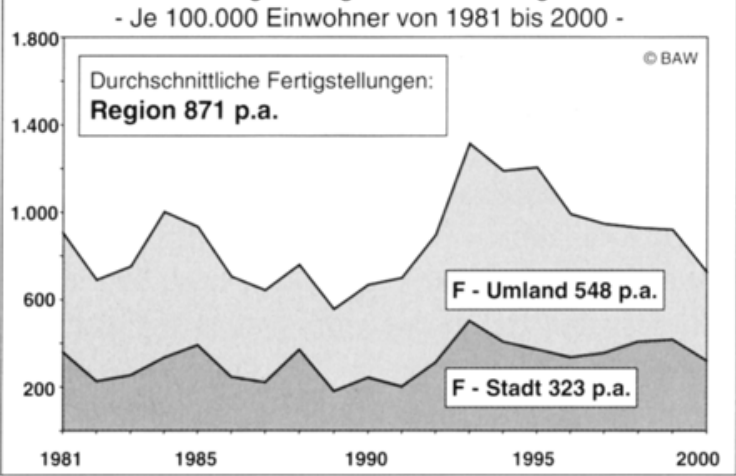

\section{Region Hannover}

Baufertigstellungen von Wohnungen - Je 100.000 Einwohner von 1981 bis 2000 -

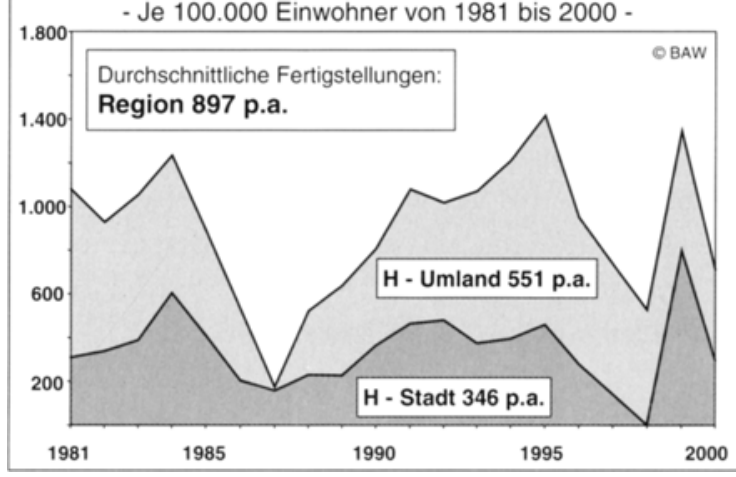

\section{Region Stuttgart}

Baufertigstellungen von Wohnungen - Je 100.000 Einwohner von 1981 bis 2000 -

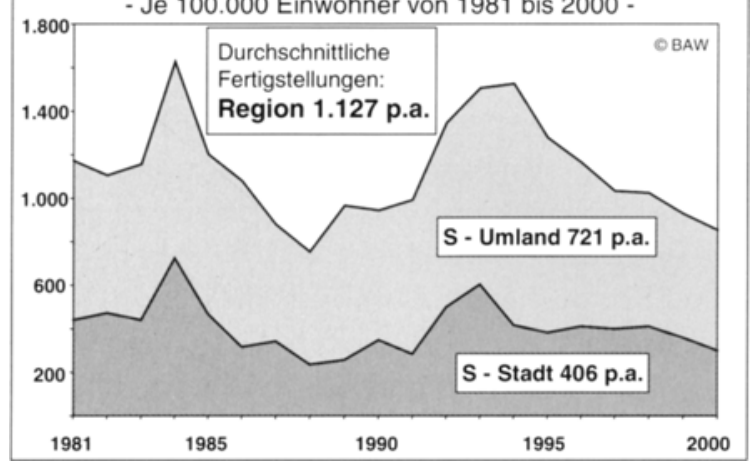

\section{Durchschnittliche Fertigstellungen pro Jahr}

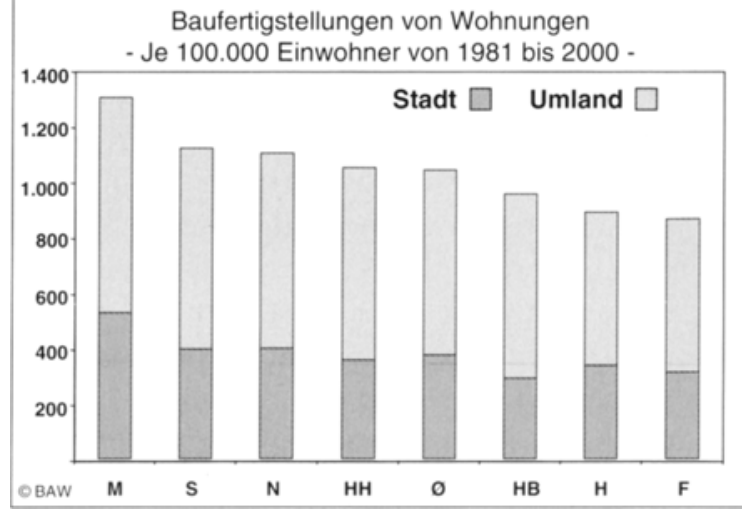

\section{Region Nürnberg}

Baufertigstellungen von Wohnungen - Je 100.000 Einwohner von 1981 bis 2000 -

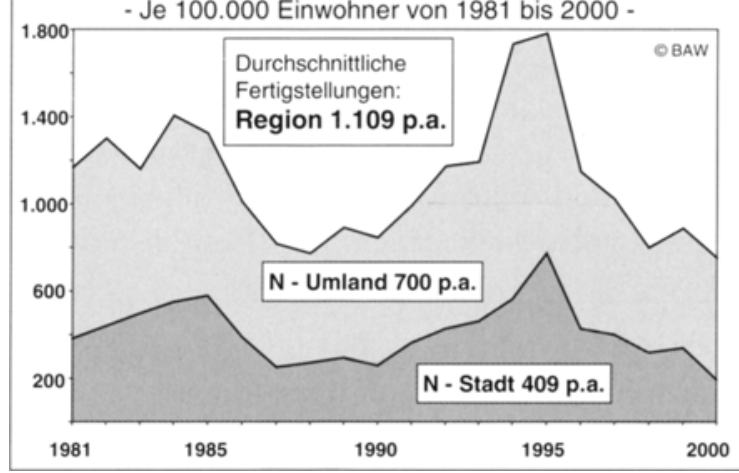

\section{Region München}

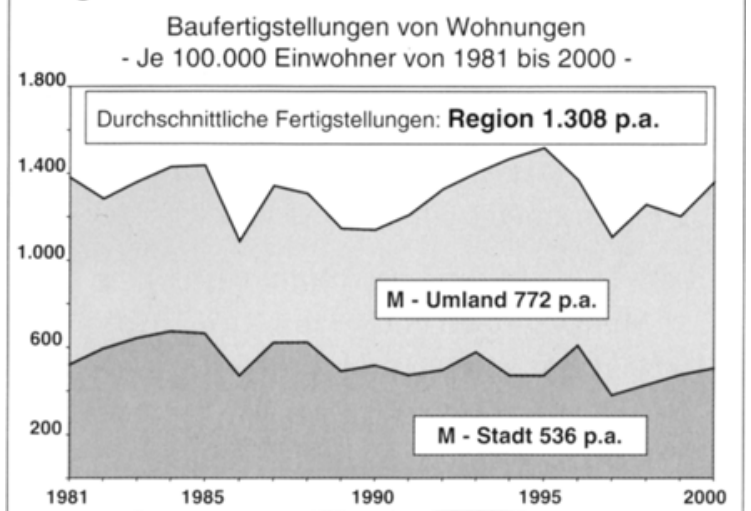

Quelle: Statistisches Bundesamt, Statistische Landesämter, Statistisches Jahrbuch deutscher Gemeinden, eigene Berechnungen 
Abbildung 4 Baufertigstellungen von Ein- und Zweifamilienhäusern in den Großstadtregionen 1981-2000
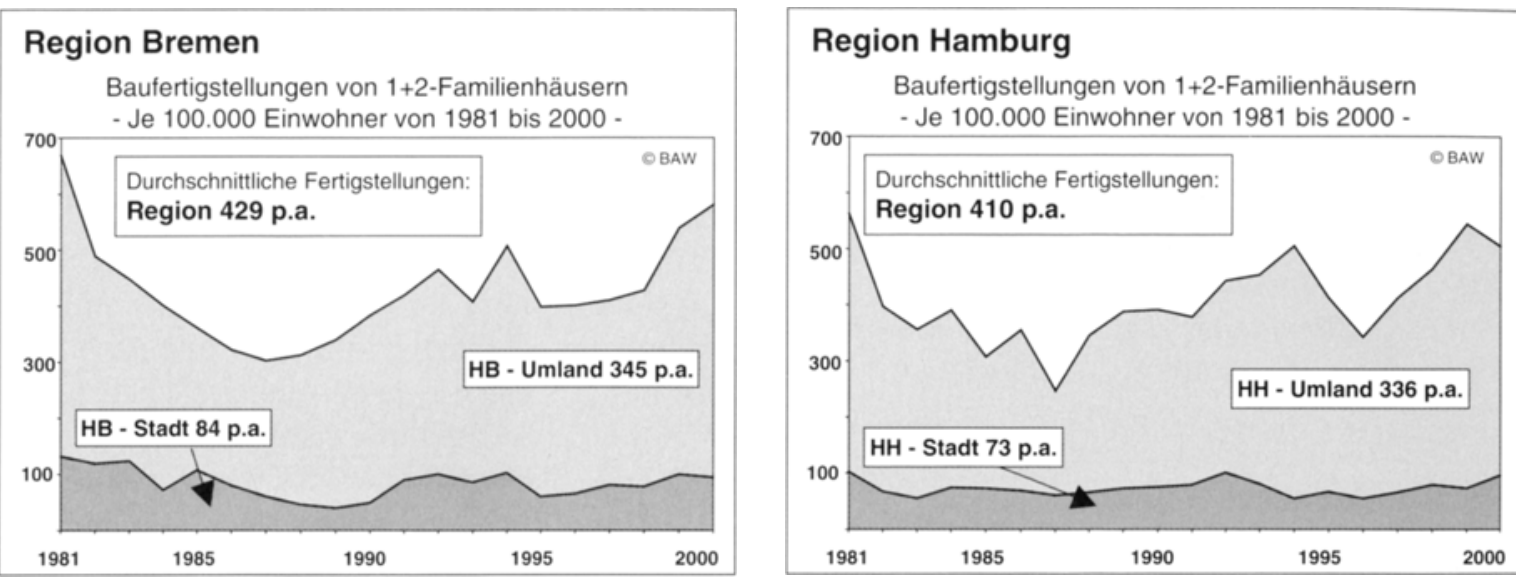

\section{Region Frankfurt am Main}

Baufertigstellungen von 1+2-Familienhäusern - Je 100.000 Einwohner von 1981 bis 2000 .

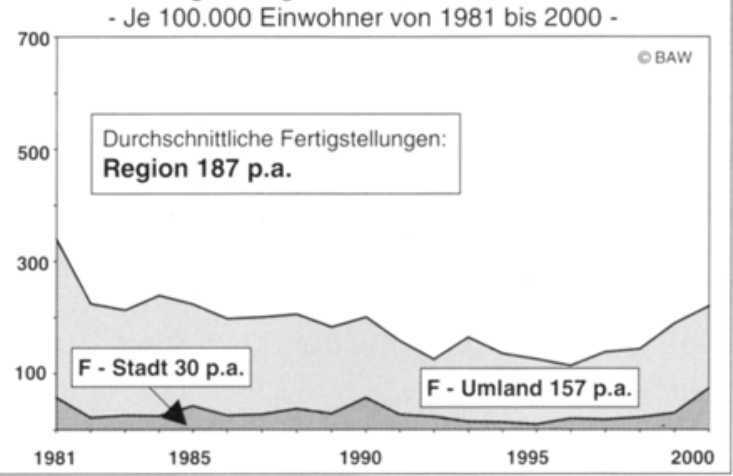

\section{Region Stuttgart}

Baufertigstellungen von 1+2-Familienhäusern - Je 100.000 Einwohner von 1981 bis 2000 .



Durchschnittliche Fertigstellungen pro Jahr

Baufertigstellungen von 1+2-Familienhäusern - Je 100.000 Einwohner von 1981 bis 2000 .

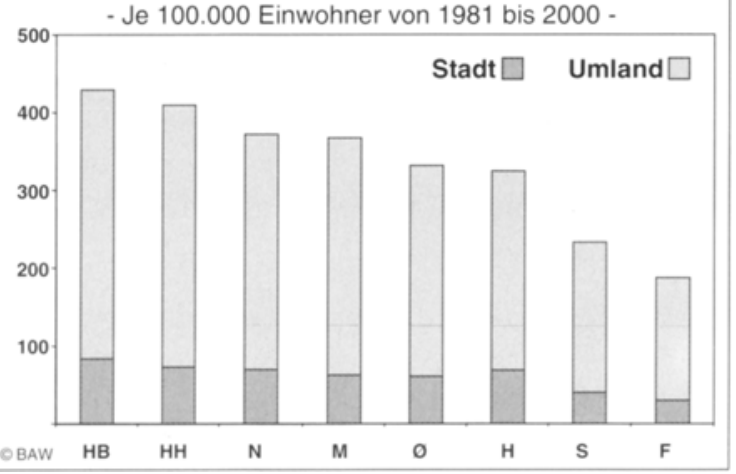

\section{Region Hannover}

Baufertigstellungen von 1+2-Familienhäusern

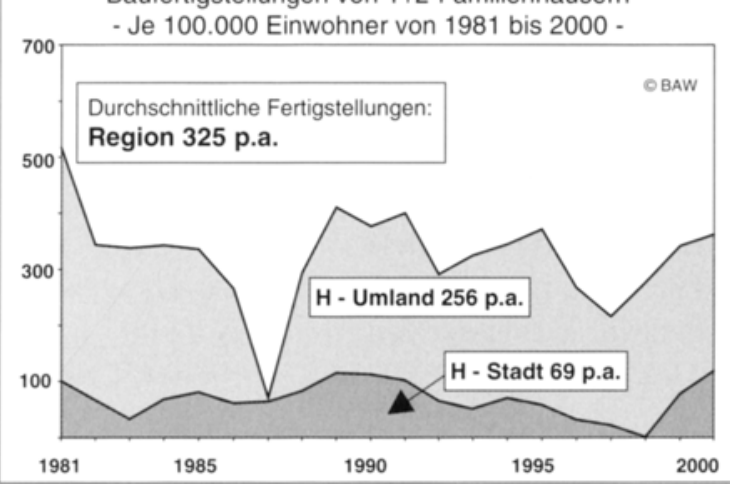

Region Nürnberg

Baufertigstellungen von 1+2-Familienhäusern

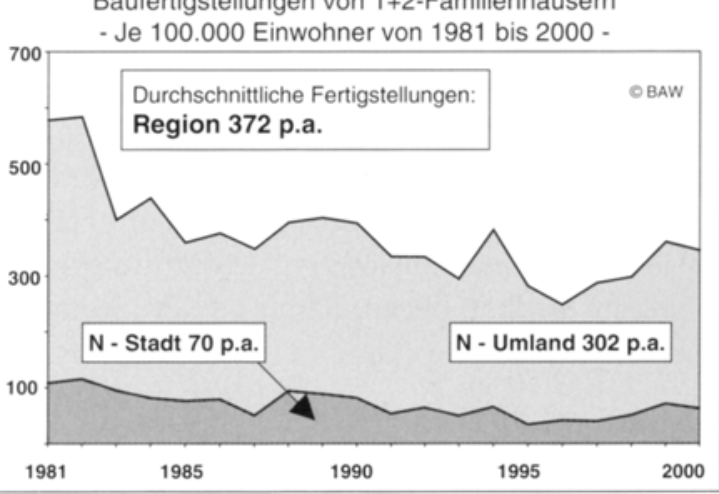

Region München

Baufertigstellungen von 1+2-Familienhäusern - Je 100.000 Einwohner von 1981 bis 2000

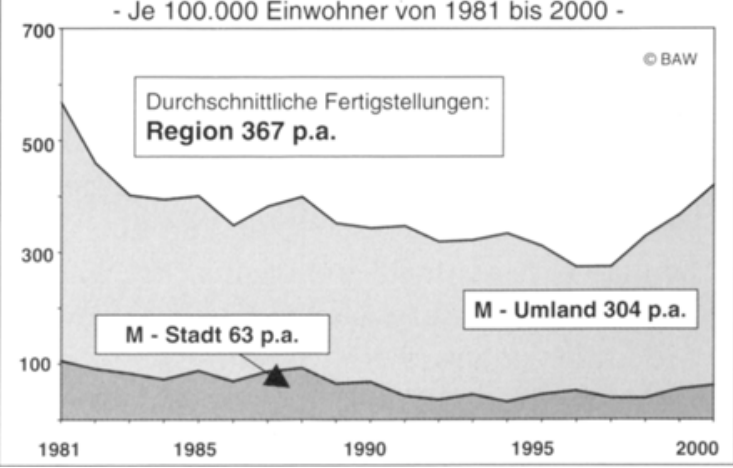

Quelle: Statistisches Bundesamt, Statistische Landesämter, Statistisches Jahrbuch deutscher Gemeinden, eigene Berechnungen 
Interessant ist aber die Entwicklung seit Mitte der 90er Jahre, die entgegen dem allgemeinen Trend bei der Erstellung von Wohnungen zu sehen ist: So ziehen die Baufertigstellungen der Ein- und Zweifamilienhäuser seit 1996 - sowohl im Umland als auch in der Stadt wieder deutlich an, während die allgemeinen Baufertigstellungen von Wohnungen eher rückläufig waren. Offensichtlich hat es in der zweiten Hälfte der 90er Jahre ein starkes Aufleben des Gebäudetyps Ein- und Zweifamilienhaus in den Stadtregionen gegeben.

\section{Zum Stadt-Umland-Verhältnis der Baufertigstellungen}

Unter dem Aspekt der Suburbanisierung der Bevölkerung sind die Verhältnisse der Baufertigstellungen zwischen Zentrum und Umland besonders zu beachten. Die im vorigen Abschnitt dargestellten Analysen zu den Baufertigstellungen werden nun auf ihr Stadt-Umland-Verhältnis konzentriert. Abbildung 5 zeigt die Ergebnisse der Baufertigstellungen von Wohnungen und der Ein- und Zweifamilienhäuser im Verhältnis von Stadt und Umland für die Jahre 1981-2000. Dabei wird - wie in den vorangegangenen Analysen auch - nach dem Entwicklungsverlauf und dem jährlichen Durchschnittswert unterschieden.

Über 20 Jahre betrachtet, hat im jährlichen Durchschnitt das Zentrum von Bremen bei der Erstellung von Wohnungen am schwächsten abgeschnitten. Das Verhältnis von $1: 2,2$ bedeutet, dass bei der Fertigstellung einer Wohnung je 100000 Einwohner im Zentrum gleichzeitig mehr als doppelt so viele Wohnungen im Umland geschaffen worden sind. Anders ausgedrückt könnte man aus Sicht der Gesamtregion auch folgern, dass von allen sieben analysierten Verdichtungszentren die Stadt Bremen ihrem Umland am weitestgehenden die Wohnungsbauaktivitäten überlassen hat.

Schließlich fallen die Stadt-Umland-Verhältnisse in den anderen Regionen teilweise deutlich geringer aus. So werden - über 20 Jahre gesehen - im Münchner Umland lediglich 1,4 neue Wohnungen je 100000 Einwohner gebaut, wenn im Zentrum eine neue Wohnung fertig gestellt wird. Im Mittelwert aller sieben Regionen liegt dieses Verhältnis bei $1: 1,7$.

Demgegenüber fallen die Stadt-Umland-Verhältnisse bei der einwohnerbezogenen Erstellung von Ein- und Zweifamilienhäusern deutlich größer aus. Im Mittelwert liegt das Verhältnis dieses Gebäudetyps bei $1: 4,4$. Besonders deutlich fallen diese Verhältnisse in den Regionen Frankfurt $(1: 5,3)$, aber auch München und Stuttgart (jeweils $1: 4,8$ ) aus. Dieses Ergebnis bedeutet aus der einwohnerbezogenen Sichtweise, dass beim
Neubau eines Hauses in der Kernstadt gleichzeitig im Umland etwa fünf Mal Richtfest gefeiert werden kann. In Relation dazu sind die Verhältnisse in Bremen $(1: 4,1)$ und Hannover ${ }^{18}(1: 3,7)$ deutlich geringer ausgeprägt.

In der zeitlichen Entwicklung der Stadt-Umland-Verhältnisse zeigen sich zunächst wesentlich geringere Schwankungen bei der Erstellung von Wohnungen gegenüber den Aktivitäten im Ein- und Zweifamilienhausbau. Offensichtlich besteht zwischen Stadt und Umland bei der Erstellung von Wohnungen eine recht große Entwicklungsparallelität. Demgegenüber lassen sich bei dem Haustyp Ein- und Zweifamilienhaus anhand der Zeitreihen sehr viel stärkere Unterschiede identifizieren. So ist im Durchschnitt aller analysierten Städte das Stadt-Umland-Verhältnis bei diesem Haustyp in den 90er Jahren deutlich stärker zu Gunsten des Umlandes ausgeprägt, als dies noch in den 80er Jahren der Fall war.

Die regionalen Besonderheiten sind den Teilabbildungen zu entnehmen. So fallen die besonders groß ausgeprägten Verhältnisse in der ersten Hälfte der 90er Jahre in Frankfurt und München ins Auge. Ein Abgleich mit der vorhergehenden Abbildung 4 zeigt, dass es sich hier allerdings weniger um besonders starke Aktivitäten im Umland handelt, als vielmehr dem relativ stärkeren Rückzug der Verdichtungszentren bei der Fertigstellung von Ein- und Zweifamilienhäusern. Bei der jüngeren Entwicklung sind auch in den Zentren wieder vermehrt Baufertigstellungen dieses Gebäudetyps festzustellen, sodass sich die Verhältnisse wieder verkleinert haben.

\section{Fazit: Regionalpolitische Relevanz der empirischen Ergebnisse}

Die empirischen Ergebnisse in den sieben analysierten Stadtregionen zeigen große Gemeinsamkeiten in den Entwicklungstendenzen der Suburbanisierung und den Bauaktivitäten auf. Alle Städte verlieren per Saldo jedes Jahr etwa 0,5\% ihrer Bevölkerung an das sie umgebende Umland. Über die beobachteten 20 Jahre summieren sich diese Einwohnerverluste auf etwa $10 \%$ auf, wobei durchaus regionale Unterschiede existieren. So hat Nürnberg mit ca. 7,5\% seiner Gesamtbevölkerung deutlich weniger Einwohner an das Umland abgeben müssen, als dies in Frankfurt am Main mit ca. $12 \%$ der Fall gewesen ist. 
Abbildung 5 Stadt-Umland-Verhältnis der Baufertigstellungen 1981-2000
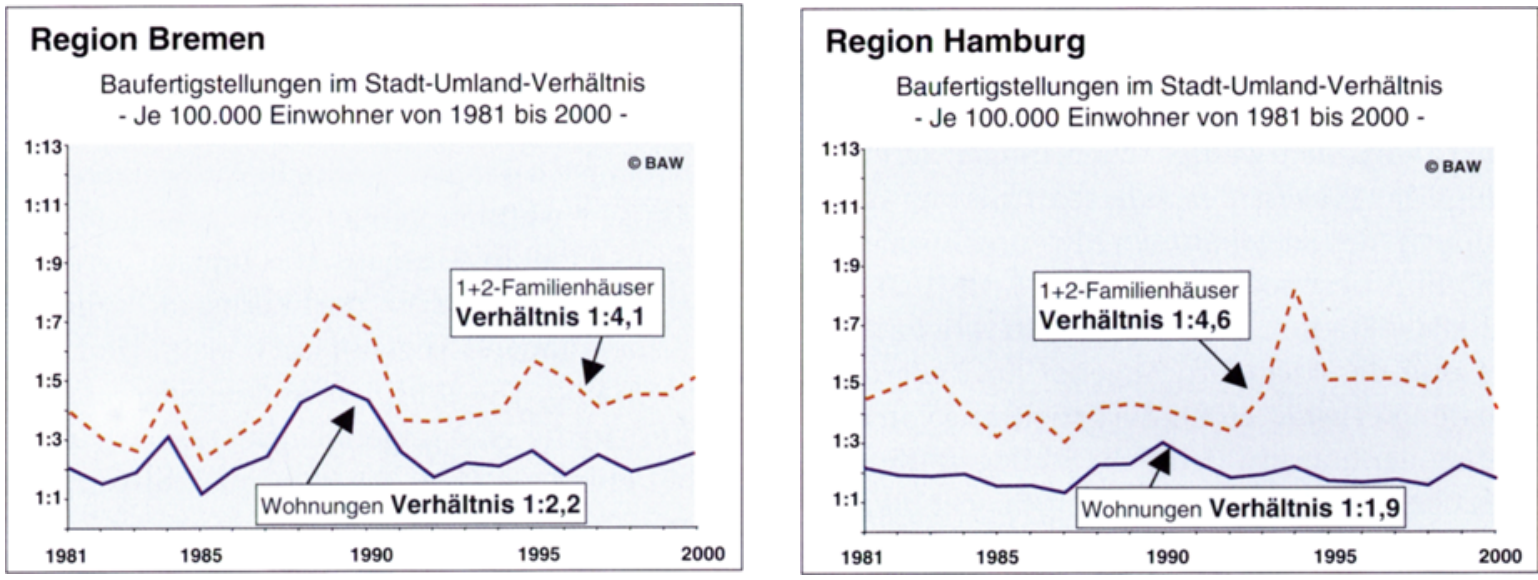

\section{Region Frankfurt am Main}

Baufertigstellungen im Stadt-Umland-Verhältnis - Je 100.000 Einwohner von 1981 bis 2000 .

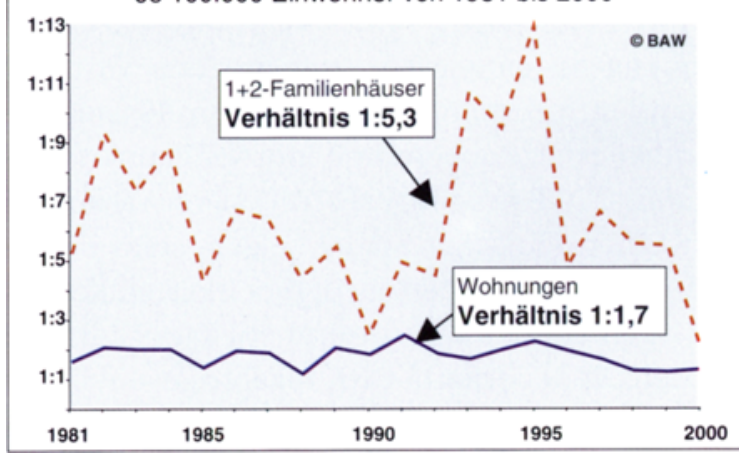

\section{Region Hannover}

Baufertigstellungen im Stadt-Umland-Verhältnis - Je 100.000 Einwohner von 1981 bis 2000 -

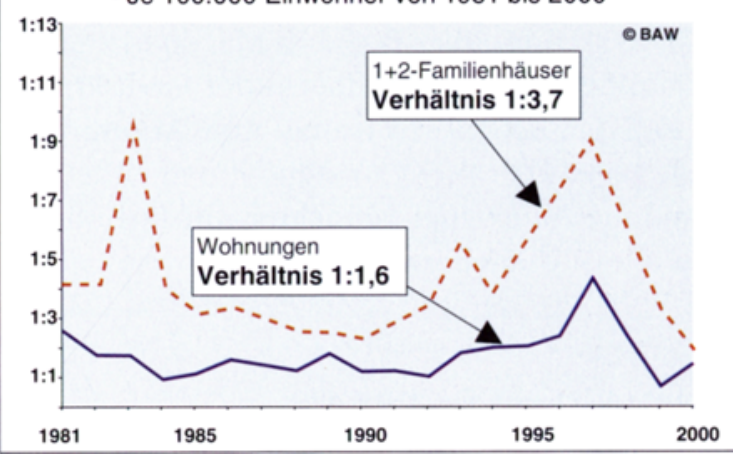

\section{Region Stuttgart}

Baufertigstellungen im Stadt-Umland-Verhältnis - Je 100.000 Einwohner von 1981 bis 2000

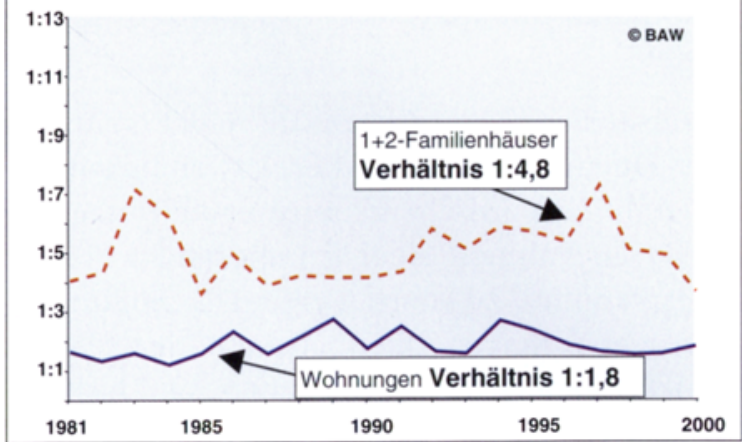

\section{Durchschnittliche Fertigstellungen pro Jahr}

Baufertigstellungen im Stadt-Umland-Verhältnis - Je 100.000 Einwohner von 1981 bis 2000

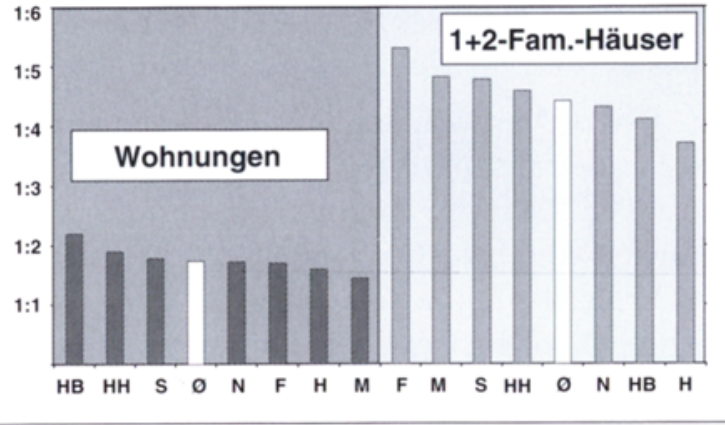

\section{Region Nürnberg}

Baufertigstellungen im Stadt-Umland-Verhältnis - Je 100.000 Einwohner von 1981 bis 2000 .
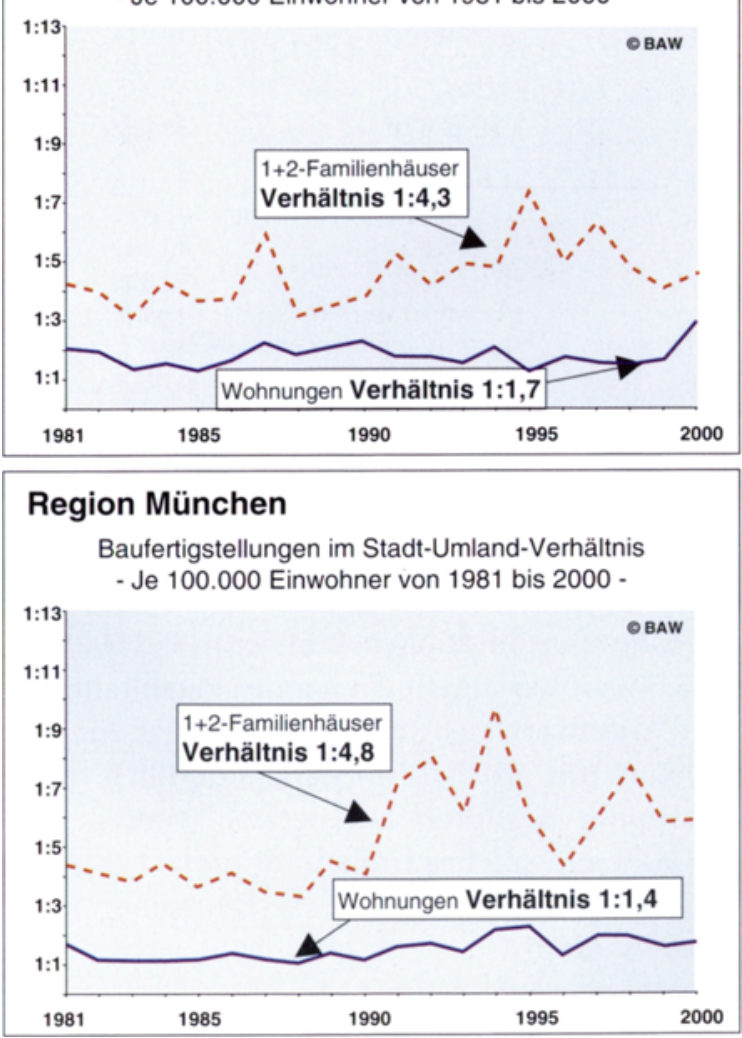

Quelle: Statistisches Bundesamt, Statistische Landesämter, Statistisches Jahrbuch deutscher Gemeinden, eigene Berechnungen 
Die Entwicklungstendenzen der Suburbanisierung üben einen starken Einfluß auf das Verhältnis der einwohnerbezogenen Baufertigstellungen aus, wobei die Kernstadt gegenüber dem Umland mit einem Verhältnis von $1: 1,7$ deutlich weniger Wohnungen auf den Markt bringt. Im Prozess der Suburbanisierung spielt der Gebäudetyp des so genannten Ein- und Zweifamilienhauses eine besondere Rolle. Die Untersuchung macht in allen Städten die höhere Attraktivität des Umlandes und das knappere Angebot an Baugrundstücken in den Kernstädten für die Errichtung von Einund Zweifamilienhäusern deutlich: So liegen die einwohnerbezogenen Baufertigstellungen ca. vier bis fünf Mal höher als im Zentrum.

Dabei sind regionale Besonderheiten und die Baustruktur im Bestand der Städte zu berücksichtigen. Wie der Tabelle 1 zu entnehmen ist, verfügen die Einwohner der Stadt Bremen über fast drei Mal so viele Einund Zweifamilienhäuser ${ }^{19}$, als dies in der Stadt Frankfurt der Fall ist. Die Ausstattung mit Wohnungen weicht demgegenüber nicht so stark voneinander ab. Eine Ausnahme bildet hier München mit fast 58000 Wohnungen je 100000 Einwohnern.

Tabelle 1

Bestand je 100000 Einwohner (31.12.2000)

\begin{tabular}{|lcc|}
\hline Stadt & $\begin{array}{c}\text { Ein- und } \\
\text { Zweifamilienhäuser }\end{array}$ & Wohnungen \\
\hline Bremen & 15081 & 52130 \\
Hamburg & 8746 & 50076 \\
Nürnberg & 8697 & 52192 \\
& & \\
Ø-Wert & $\mathbf{8 0 4 6}$ & $\mathbf{5 2 7 9 2}$ \\
Hannover & 6699 & $\mathbf{5 4 9 8 8}$ \\
München & 6019 & 57941 \\
Stuttgart & 5801 & 49591 \\
Frankfurt & 5280 & 52630 \\
\hline
\end{tabular}

Quelle: Statistisches Bundesamt, eigene Berechnungen

Für derartige Unterschiede sind - neben vielen anderen Faktoren - die Bau- und Bodenpreise ein wesentlicher und determinierender Faktor. So weist der Ring Deutscher Makler (RDM) im 1. Quartal 2001 für Bremen einen durchschnittlichen Preis von 355000 DM für ein 125 qm großes und freistehendes Eigenheim bei mittlerem Wohnwert aus. In Frankfurt muss für das gleiche Objekt mit 770000 DM bereits deutlich mehr als der doppelte Kaufpreis ausgegeben werden. Am teuersten wäre ein solches Haus in München, wo mit 925000 DM die Millionengrenze (in Deutsche Mark) fast erreicht wird.

\section{Welche Schlüsse sind aus den Ergebnissen zu ziehen?}

Aus der Stadt Fortziehende versuchen zunächst, ihre Vorstellungen in der Nähe des bisherigen Wohnstandortes zu realisieren. ${ }^{20}$ Befragungen in Münster ${ }^{21}$ zeigen, dass über $50 \%$ der aus der Stadt Abgewanderten lieber in Münster wohnen geblieben wären. Eine Untersuchung in Hamburg ${ }^{22}$ ergab, dass umzugsbereite Haushalte sich zunächst in der Kernstadt umschauten, dann aber mangels Angebot doch in das Umland abgewandert sind. Die Beispiele sollen zeigen, dass auch die Kernstädte eine angebotsorientierte Baulandpolitik betreiben müssen, die den qualitativen Anforderungen ihrer Einwohner an hochwertigen Wohnraum und attraktiven Baugrundstücken gerecht wird.

Die Herausforderung für die Städte besteht vor allem darin, standortgebundene Qualitätskonzepte (z.B. „Wohnen am Wasser") mit einem unverwechselbaren Charakter zu entwickeln, um mit den Vorteilen des Umlandes (z.B. mehr Wohnfläche pro Person, größere Grundstücke, geringere Bodenpreise) konkurrieren zu können. Wie Befragungen ${ }^{23}$ in den Verwaltungen verschiedener deutscher Städte zeigen, steht das Thema Stadt-Umland-Wanderung in den meisten Kommunen weit oben auf der Tagesordnung. Viele Städte erarbeiten zielgruppenorientierte Konzepte gegen die Abwanderung der Bevölkerung. Die in der jüngeren Entwicklung zu beobachtenden Aktivitäten der Städte zur Erstellung von Ein- und Zweifamilienhäusern könnte als Versuch zum Halten von Einwohnern mit höheren Einkommen innerhalb der Stadtgrenzen interpretiert werden.

Grundsätzlich zeigen die empirischen Erkenntnisse im Stadt-Umland-Verhältnis die Notwendigkeit auf, die Baulandpolitik zwischen Zentrum und Umland stärker - etwa im Rahmen einer gemeinsamen Flächennutzungsplanung - zu koordinieren. Die "Sollbruchstelle“ derartiger Vorhaben, die über reine Absichtserklärungen zur Kooperation hinaus gehen, sind bisher häufig die fiskalischen Effekte, die durch die Stadt-UmlandWanderung der Bevölkerung einhergehen. So ist z. B. im Stadtstaat Bremen von einem jährlichen Steuerausfall in Höhe von etwa $3300 €$ je Einwohner ${ }^{24}$ auszugehen, sofern dieser in der Umlandkommune den ersten Wohnsitz nimmt.

Die weitere Entwicklung der Stadt-Umland-Wanderungen der Bevölkerung ist derzeit nur schwer zu beurteilen. Manche Autoren ${ }^{25}$ vermuten wegen der aktuellen Abschwächung der Suburbanisierung und der Einschätzung wohnungspolitischer Rahmenbedingungen einen weiteren Rückgang der Suburbanisierungsverluste im Sinne einer Trendumkehr zu Gunsten der Städte. 
Derartige Aussagen über die Veränderung der nunmehr viele Jahrzehnte anhaltenden Suburbanisierungsprozesse sind m.E. noch zu früh. Dazu bedarf es der weiteren Beobachtung der Stadt-Umland-Wanderungen insbesondere unter den Bedingungen einer wieder verbesserten wirtschaftlichen Gesamtsituation. Schließlich würde dadurch die Investitionsbereitschaft privater Haushalte in die Qualitätssteigerung ihrer Wohnverhältnisse und damit die regionale Umzugsbereitschaft wieder zunehmen. In diesem Fall könnte das Umland - auf Grund der in diesem Beitrag gezeigten recht stabilen Stadt-Umland-Verhältnisse bei der Erstellung von Wohnungen sowie von Ein- und Zweifamilienhäusern - ein größeres und vermutlich auch differenzierteres Immobilienangebot für potenzielle Nachfrager bereitstellen.

Unterstützend wirkt sich dabei der bereits weit voran geschrittene Ausdifferenzierungsprozess der Wohnungsmärkte in den Umlandregionen aus. Dieser Reifeprozess, der sich in mehreren Jahrzehnten Suburbanisierung vollzogen hat, hat zu einer größeren Eigenständigkeit und Angebotsvielfalt bei der Infrastruktur der Umlandkommunen geführt. ${ }^{26}$ Dadurch weisen diese Räume eine hohe Attraktivität u.a. für überregionale Zuwanderer auf, die beispielsweise zur Aufnahme einer neuen beruflichen Tätigkeit von außen in die Gesamtregion kommen. Möglicherweise nehmen diese Personen ihren Wohnsitz direkt in der Umlandregion und tragen somit - neben den aus dem Zentrum Zuziehenden - ebenfalls zu einem Einwohnerzuwachs im Umland bei. Dieser Zusammenhang wird vermutlich besonders in dynamischen Agglomerationen zum Tragen kommen, da attraktiver Wohnraum in der Kernstadt knapp ist und nur zu relativ hohen Preisen angeboten wird. Zu einer größeren Unabhängigkeit der Umlandkommunen vom Zentrum trägt allerdings nicht nur die Entwicklung auf dem Wohnungsmarkt bei. Darüber hinaus sind seit längerem relative Bedeutungsverschiebungen zwischen den Zentren und dem Umland bei mehreren Beschäftigungsindikatoren zu Gunsten der Umlandregionen festzustellen. ${ }^{2 ?}$

Für die Raumforschung wird in Zukunft die Berücksichtigung großräumiger Disparitäten, wie die Entwicklung der sog. Nord-Süd- bzw. Ost-West-Gefälle, ebenfalls eine größere Relevanz erhalten. ${ }^{28}$ So ist auf Grund der allgemeinen demographischen und wirtschaftlichen Entwicklung zu erwarten, dass die Wohnflächennachfrage in Wachstumsregionen auch weiterhin steigen wird. ${ }^{29}$ Demgegenüber wird es in strukturschwachen Regionen mit deutlichen Bevölkerungsrückgängen zunehmende Probleme mit der Platzierung des Wohnungsbestandes am Markt geben. ${ }^{30}$
Insofern sind zukünftige Analysen noch kleinräumiger und regional spezifischer als bisher zu gestalten, um den neuen regionalpolitischen Herausforderungen auch in Zukunft gerecht zu werden. ${ }^{31}$

\section{Anmerkungen}

(1)

Vgl. dazu Kloas, J;; Kuhfeld, H. (2003): Entfernungspauschale: Bezieher hoher Einkommen begünstigt - Aktuelle Ergebnisse zum Verkehrsverhalten privater Haushalte. In: DIW-Wochenbericht Nr. 42/2003, S. 623-629

(2)

Vgl. dazu folgende Fallstudien: GEWOS (1996) (Hrsg.): Umlandwanderungen in der Region Bremen, Hamburg; Hinrichs, W. (1999): Wohnsuburbanisierung am Beispiel Berlin. Ein Erklärungsrahmen (Wissenschaftszentrum Berlin für Sozialforschung FS III 99-401), Berlin; Landeshauptstadt Hannover (1994) (Hrsg.): Wanderungen zwischen Stadt und Landkreis Hannover. Entscheidungsgründe und Wohnerfahrungen. Schriften zur Stadtentwicklung 68, Hannover; Stadt Dortmund (1998) (Hrsg.): „Bilanzen“. Wohnortwechsel und Wegzugsgründe. Dortmunder Statistik, Dortmund; Stadt Karlsruhe (1998) (Hrsg.): Motive der Stadt-Umland-Wanderung in der Region Karlsruhe 1997. Ergebnisse einer Befragung zu den Wanderungsmotiven der Fortgezogenen von Karlsruhe in die Region. Beiträge zur Stadtentwicklung Nr. 6, Karlsruhe; Landeshauptstadt München (2002) (Hrsg.): Raus aus der Stadt? Untersuchung der Motive von Fortzügen aus München in das Umland 1998-2000 (Gutachten bearbeitet vom IMU-Institut für Medienforschung und Urbanistik, München), München.

(3)

Vgl. z.B. Brake, K.; Dangschat, J. S.; Herfert, G. (2002) (Hrsg.): Suburbanisierung in Deutschland - Aktuelle Tendenzen, Leske + Budrich, Opladen oder Bundesamt für Bauwesen und Raumordnung (Hrsg.): Perspektiven der Wohnungsmärkte, Informationen zur Raumentwicklung, Heft 3.2002. - Bonn

(4)

Vgl. Böltken, F.; Schneider, N.; Spellerberg, A. (1999): Wohnen Wunsch und Wirklichkeit, Subjektive Prioritäten und subjektive Defizite als Beitrag zur Wohnungsmarktbeobachtung. In: Informationen zur Raumentwicklung, H. 2/1999, S. 141-156

(5)

Derartige Tendenzen finden sich auch in ostdeutschen Regionen, die insgesamt starken Bevölkerungsverlusten unterliegen; vgl. z.B. Herfert, G. (2002): Disurbanisierung und Reurbanisierung - Polarisierte Raumentwicklung in der ostdeutschen Schrumpfungslandschaft. In: Raumforschung und Raumordnung, H. 5-6, S. 334-344

(6)

Vgl. Frankfurter Allgemeine Zeitung 01.08.2003, S. 41

(7)

Vgl. Bundesamt für Bauwesen und Raumordnung (2000) (Hrsg.): Raumordnungsbericht 2000 (Berichte BBR 7), Bonn 
(8)

Vgl. Kühn, M. (2001): Regionalisierung der Städte - Eine Analyse von Stadt-Umland-Diskursen räumlicher Forschung und Planung. In: Raumforschung und Raumordnung, Heft 5-6, S. $402-$ 411

(9)

Vgl. Reiss-Schmidt, S. (2003): Herausforderungen und Chancen kooperativer Regionalentwicklung - Perspektiven für die Region München. In: DISP 152, S. 71-79

\section{(10)}

Vgl. zur Diskussion der verschiedenen Facetten der Suburbanisierung beispielsweise die Beiträge in dem Sammelband „Suburbanisierung in Deutschland - Aktuelle Tendenzen“. Brake, K.; Dangschat, J. S.; Herfert, G. (2001) (Hrsg.): Leske + Budrich, Opladen

\section{(11)}

Vgl. die Literaturauswertung in Spiekermann, K. (1992): Reurbanisierung der Bevölkerung? In: RaumPlanung 59, S. 215-226

(12)

Wesentliche Ergebnisse wurden auf dem 40. Winterseminar der Gesellschaft für Regionalforschung am 28. Februar 2003 in Igls (Tirol) zur Diskussion gestellt. Für kritische Anmerkungen der Seminarteilnehmer, insbesondere des Diskussionsleiters Herrn Dr. Hansjörg Bucher vom Bundesamt für Bauwesen und Raumordnung (BBR) aus Bonn, möchte ich mich herzlich bedanken.

(13)

Für die Region Stuttgart konnten nur 13 Einzelwerte (teilweise über mehrere Jahre aggregierte Datensätze) in die Untersuchung eingehen. Ich danke dem Verband Region Stuttgart und dem Bundesamt für Bauwesen und Raumordnung, Bonn für die Überlassung der Daten.

(14)

Zwischen den Wanderungsdaten der Statistischen Landesämter und denen aus der Kommunalstatistik existieren in manchen Städten starke Abweichungen. Im 20-Jahreszeitraum sind Differenzen von bis zu $35 \%$ (alle Zeitpunkte aggregiert) festzustellen.

(15)

Die jährlichen Angaben zu Hannover sind erklärungsbedürftig. So beruhen die Schwankungen 1998 und 1999 nach Aussagen des Statistischen Amtes der Stadt Hannover auf der verspäteten Angabe der Baufertigstellungen, d.h. die 98er Zahlen sind in den 99er Zahlen enthalten. Ebenfalls keine Werte lagen offensichtlich für $1987 \mathrm{im}$ Umland Hannover vor.

(16)

Einbezogen sind hier alle Baufertigstellungen, also auch Baumaßnahmen an bestehenden Gebäuden.

(17)

Im weiteren Text wird dafür der allgemein geläufige Begriff des Ein- bzw. Zweifamilienhauses verwendet, auch wenn diese Objekte nicht nur von Familien (z.B. von Paaren ohne Kinder) nachgefragt werden.
(18)

Zur besseren Lesbarkeit der Teilabbildung „Region Hannover“ sind - auf Grund mangelhafter Angaben für die Jahre 1987 und 1998 - die Stadt-Umland-Verhältnisse für diese zwei Zeitpunkte in den Zeitreihen geschätzt worden; siehe dazu auch Abb. 3 und 4 .

(19)

Charakteristisch in Bremen ist u.a. der Gebäudetyp des sog. Bremer Hauses, das im Prinzip als Reihenhaus konzipiert ist. Dieser Haustyp gilt in der Statistik auch als Ein- und Zweifamilienhaus bzw. als Gebäude mit einer oder zwei Wohnungen.

(20)

Vgl. auch Schönert, M.; Wehling, W. (2000): Einwohnerentwicklung Bremerhaven (BAW-Gutachten im Auftrag Bremerhavens). - Bremen

(21)

Hauff, T. (2001): Wanderungsumfrage 2000 Münster - Fortbestand oder Differenzierung der traditionellen Wanderungsmuster. In: Stadtforschung und Statistik 2/01, S. 39-43

(22)

Empirica (2000) (Hrsg.): Stadtentwicklungspolitik und Demographie - Möglichkeiten der Strukturbeeinflussung durch Städtebau und Wohnungsbau (unveröffentl. Gutachten). - Bonn, Berlin

Vgl. die Recherchen von Salden, J. (2001): Einwohnerentwicklung deutscher Städte im Vergleich. In: Statistische Monatsberichte Bremen, H. 9/10, S. 185-200

(24)

Der Wert ist für das Jahr 1999 berechnet und schließt die stadtstaatenspezifischen Zuweisungen aus dem Länderfinanz ausgleich; vgl. Pohl, M. (2001): Fiskalische Effekte von Arbeitsplätzen (BAW-Diskussionsbeiträge 2), Bremen; vgl. für die Region Hamburg auch die Analyse von Ramms, T. (2002): Arme Stadt, reiches Land? Ein Beitrag zur fiskalischen Äquivalenz in Suburbanisierungsprozessen am Beispiel der Metropolregion Hamburg. In: Neues Archiv 1/2002, S. 71-93

(25)

Vgl. Hallenberg, B. (2002): Aktuelle Entwicklungen und Perspektiven der Stadt-Umland-Wanderung unter besonderer Berücksichtigung der Wohneigentumsbildung. In: vhw Forum Wohneigentum, H. Juni/Juli, S. 133-142

Vgl. Aring, J. (2003): Stadt-Umland-Wanderung und die Ausdifferenzierung der Wohnungsmärkte. In: vhw Forum Wohneigentum, Heft 5/2003 und Harlander, T. (2001) (Hrsg): Villa und Eigenheim - Suburbaner Städtebau in Deutschland. - Stuttgart, München 
(27)

Vgl. dazu Schönert, M. (2000): Das personelle Innovationspotenzial - Empirische Ergebnisse zur Entwicklung regionaler Disparitäten in der Bundesrepublik Deutschland (Regionalwirtschaftliche Studien 18, BAW Institut für Wirtschaftsforschung). - Bremen; Bade, F.-J.; Niebuhr, A.; Schönert, M. (2002): Spatial Structural Change: Evidence and Prospects. In: Schätzl, L.; Revilla Diez, J. (Hrsg.): Technological Change and Regional Development in Europe. - Heidelberg, New York, S. 43-60; Niebuhr, A. (2002): Die räumliche Verlagerung von Arbeitsplätzen - ein weiteres Motiv der Wohnsuburbanisierung? In: vhw Forum Wohneigentum, H. Aug.-Sept., S. 190-193 und Koller, M. (2003): Der heimliche Finanzausgleich. In: Informationen zur Raumentwicklung, H. 5, S. 271-294

\section{(28)}

Vgl. z.B. Lammers, K. (2003).: Süd-Nord-Gefälle in West- und Ostdeutschland? In: Wirtschaftsdienst, H. 11, S. 736-739; Bade, F.-J. (2003): Die regionale Entwicklung der Erwerbstätigkeit bis 2010 (Manuskript, Universität Dortmund). - Dortmund
(29)

Vgl. Bucher, H.; Schlömer, C. (2003): Der demographische Wandel und seine Wohnungsmarktrelevanz. In: vhw Forum Wohneigentum, H. Juni-Juli, S. 121-126 und Just, T, (2003): Demografie läßt Immobilien wackeln. In: Deutsche Bank Research (Hrsg,): Themen international, H. 283. - Frankfurt am Main

(30)

Vgl. Müller, B. (2003): Regionalentwicklung unter Schrumpfungsbedingungen - Herausforderung für die Raumplanung in Deutschland. In: Raumforschung und Raumordnung, H. 1-2, S. $28-42$

(31)

Siehe dazu auch die Aufsätze zum Thema „Wohnungsmarktbeobachtung - Systeme - Indikatoren - Ergebnisse“. In Informationen zur Raumentwicklung, H. 2 (1999) und Bundesamt für Bauwesen und Raumordnung (2003) (Hrsg.): Bauland- und Immobilienmärkte, Ausgabe 2003, Berichte, Bd. 16. - Bonn

Dr. Matthias Schönert

BAW Institut für Wirtschaftsforschung

Wilhelm-Herbst-Straße 5

28359 Bremen

E-Mail: m.schoenert@baw-bremen.de 